\title{
Prevalance of inducible clindamycin resistance of Staphylococcus aureus from various clinical specimens by $D$ test in tertiary care hospital
}

\author{
Dr Vyoma chudasama, Dr. Hasumati Solanki, Dr. Minesh Vadsmiya, \\ Dr M M Vegad \\ Assistant professor in Microbiology, B J Medical college, Ahmedabad; \\ Associate professor in Microbiology, B J Medical college, Ahmedabad; \\ Professor and Head of the department in Microbiology, B J Medical college, Ahmedabad;
}

\begin{abstract}
Aim:Therapeutic failure to Clindamycin has been reported due to mechanisms which confer resistance constitutively, or by the presence of low level inducers which can lead to therapeutic failure. Therefore, this study was undertaken to identify the strains that have the potential to become resistant during therapy.Materials and methods: Inducible Clindamycin resistance was tested by the Clindamycin disc induction test (D test) as per the CLSI recommendations. Results:The study showed 54.78\% MRSA isolates and 32.5\% inducible Clindamycin resistant isolates among them as compared to $15.53 \%$ in MSSA isolates. Conclusion: We concluded that routine screening for inducible resistance to Clindamycin must be performed so that the drug is used effectively and for maximum clinical utility.
\end{abstract}

Keywords : inducible clindamycin resistance, staphylococcus

\section{Introduction}

The increasing frequency of Methicillin resistant Staphylococcus aureus (MRSA) infections and the changing patterns in antimicrobial resistance have led to renewed interest in the use of macrolide-lincosamidestreptogramin B (MLSB) antibiotics to treat such infections [1]. However, their widespread use has led to an increase in the number of Staphylococcal strains which are resistant to MLSB antibiotics [2]. Resistance to MLSB can occur by two different mechanisms: an active efflux mechanism encoded by the msr A gene (macrolides streptogramin resistance) and the ribosomal target modification affecting macrolides, lincosamides and type B streptogramins coded by the erm gene (MLSB resistance). erm genes encode enzymes that confer inducible or constitutive resistance to MLS agents via methylation of the 23S rRNA, thereby reducing the binding by MLS agents to the ribosome. The msr A gene confers the so called MS phenotype (resistance to erythromycin, inducible resistance to streptogramins and susceptibility to clindamycin) by efflux [3]. MLSB resistance can be either constitutive (cMLSB) or inducible (iMLSB). In vitro Staphylococci isolates with constitutive resistance are resistant to both erythromycin (E) and clindamycin (CL), while isolates with inducible resistance are resistant to, but appear to be susceptible to clindamycin CL [1]. Inducible MLSB resistance cannot be determined by using standard susceptibility test methods including standard broth based or agar dilutions susceptibility tests. Low levels of ER are the most effective inducers of inducible MLSB resistance [2].

Uncertainty about the reliability of the susceptibility reports for clindamycin, as well as confusion over the clinical importance of this inducible resistance, has led some clinicians to avoid the use of clindamycin for Staphylococcal infections where erythromycin resistance is noted. Failure to identify MLSB may lead to the clinical failure of the CL therapy. iMLSB can be detected by a disc induction test in which a distorted ' $D$ Shaped' zone of inhibition is observed around CL if an ER disc is placed nearby (15mm) [2].

Thus, the aim of the present study was to detect the inducible clindamycin resistance in clinical isolates of Staphylococcus aureus by the disc diffusion induction test.

\section{Materials And Methods}

The present study was conducted for a period of 12 months from March-2012 to February-2013 and included a total of 230 non duplicate, consecutive isolates of S.aureus from samples of pus/wound swab, respiratory tract infections, blood, urine, body fluids, etc. The Staphylococcus aureus strains were identified by using standard microbiological procedures [4]. Antibiotic susceptibility tests were performed by the KirbyBauer disc diffusion method. Methicillin resistance was detected by using a $30 \mu \mathrm{g}$ cefoxitin disc. Staphylococcus ATCC 25923 was used as the control strain for the disc diffusion method.

To identify the MLSBi phenotype, the D-test was performed. A lawn culture of the isolate which was adjusted to 0.5 Mcfarland's concentration was made on a Mueller Hinton agar plate and discs of CL ( $2 \mu \mathrm{g})$ and 
ER $(15 \mu \mathrm{g})$ were placed at a distance of $15 \mathrm{~mm}$ (edge to edge) as per the CLSI recommendations, along with routine antibiotic susceptibility testing [5]. The disc diffusion test, based on the D test, showed four phenotypes.

D Positive (iMLSB Phenotype): Inducible resistance to Clindamycin was manifested by flattening or blunting of the CL zone adjacent to the ER disc, giving a D shape.(Fig.1)

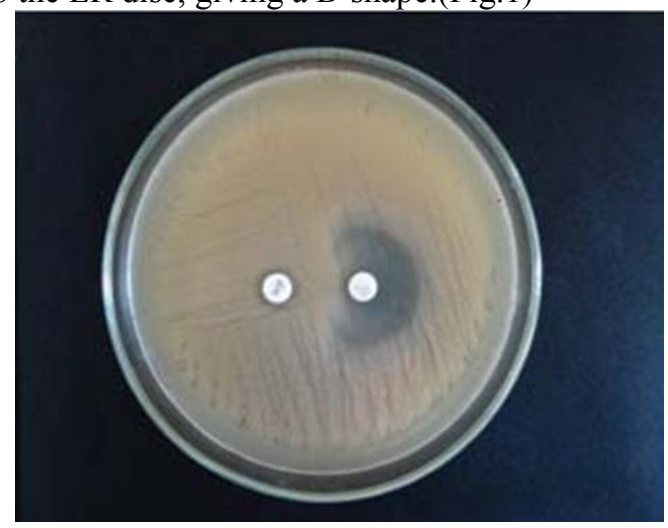

(Figure-1) D test positive isolate, inducible resistance to clindamycin

D Negative (MSB Phenotype): No flattening of the CL zone; Resistant to ER but susceptible to CL.(Fig.2)

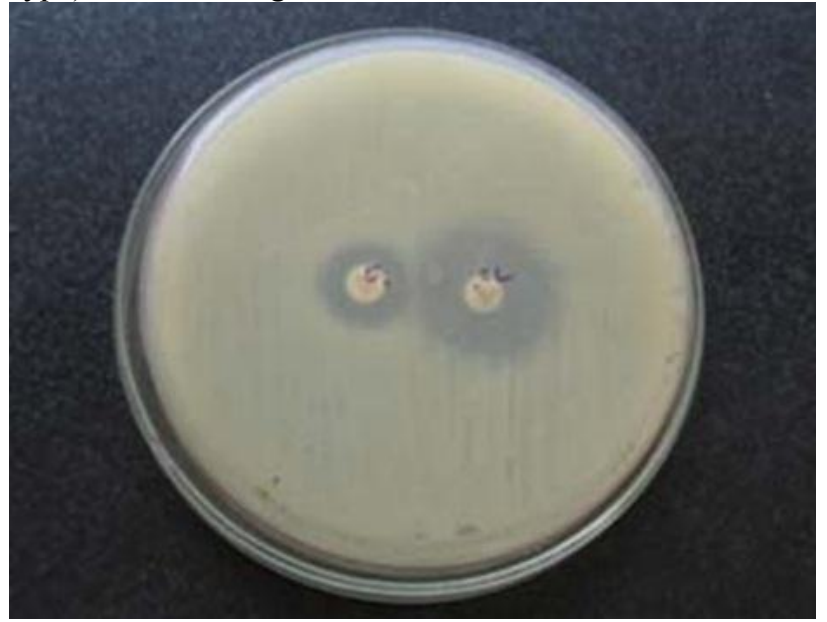

(Figure-2) D test negative isolate, resistant to erythromycin and sensitive to clindamycin

Constitutive Resistance (cMLSB Phenotype): Resistant to both ER and CL. (Fig.3)

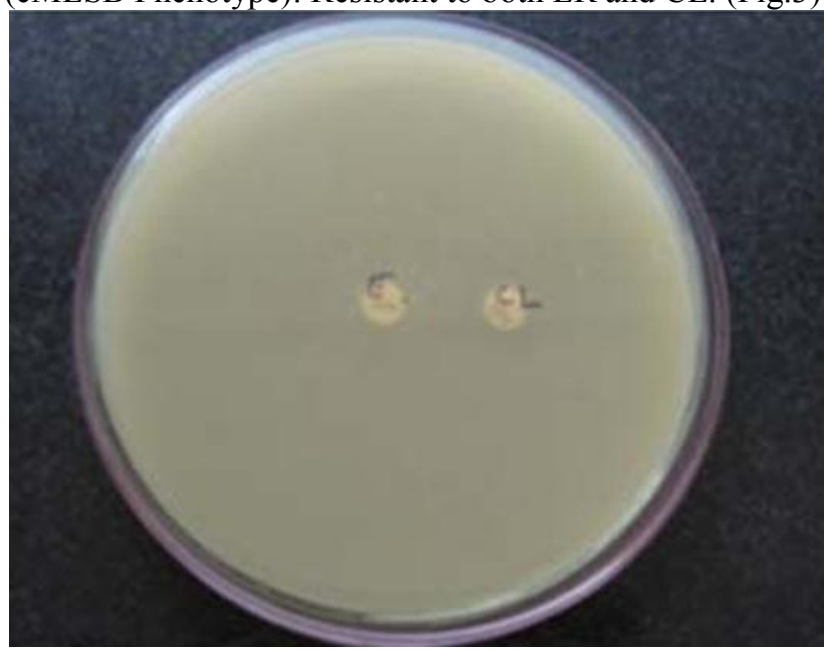

(Figure-3) Constitutive resistance to clindamycin

Sensitive (Phenotype): Sensitive to both ER and CL. (Fig.4) 


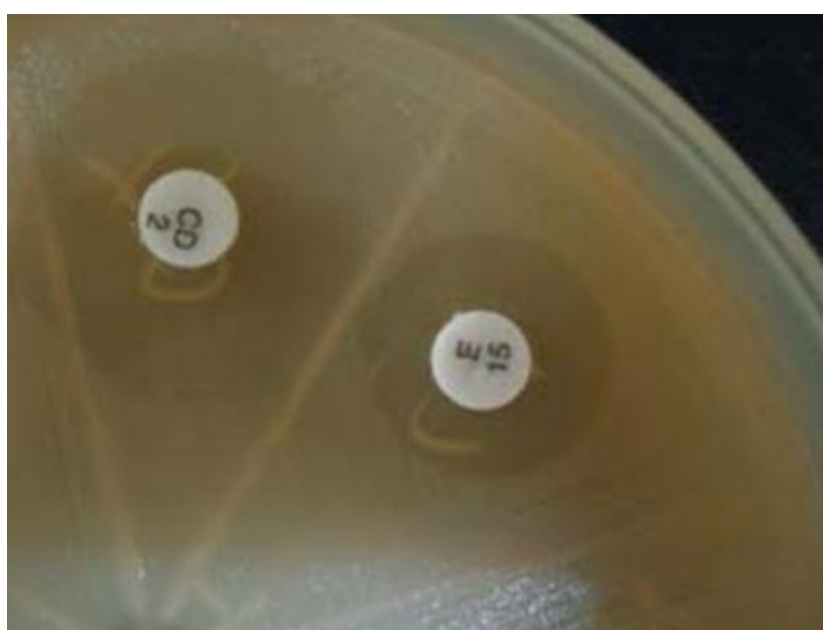

(Figure-4) Sensitive isolate. E-erythromycin $15 \mu \mathrm{g}$ disc; CL-clindamycin $2 \mu \mathrm{g}$ disc

\section{Results}

Among the 230 clinical isolates of Staphylococcus aureus, 54.78\% (126) were Methiclicin resistant Staphylococcus aureus (MRSA) and 45.21 (104) were Methicillin sensitive Staphylococcus aureus (MSSA) (Table/Fig 5) Of the 230 Staphylococcus aureus isolates, 41.3\% (95) had the E-S and CL-S phenotype, $18.26 \%(42)$ had a constitutive resistance phenotype, $15.65 \%$ (36) had an MSB phenotype and $24.89 \%$ (57) had the inducible resistance phenotype. (Table/Fig 6)Among the MRSA isolates, the inducible resistant phenotype (32.53\%) predominated over the ER-S and CL-S phenotype, the MSB phenotype and the constitutive resistance phenotype $(26.98 \%, 25.39 \%$ and $15.07 \%$ respectively). The ER-S and CL-S phenotype $(58.65 \%)$ predominated over the MSB phenotype and the inducible resistance and the constitutive resistance phenotypes $(16.34 \%, 15.38 \%$ and $9.61 \%$ respectively) among the MSSA isolates.

While in Methicillin resistant Staphylococcus aureus isolates, the constitutive CL-R phenotype level was $25.39 \%$ and the inducible résistance phenotype was $32.53 \%$; in Methicillin sensitive Staphylococcal isolates, the constitutive CL-R phenotype was $9.61 \%$ and the inducible resistant phenotype level was $15.38 \%$.

\section{Discussion}

Clindamycin is a drug which is useful for treating skin and soft tissue infections which are caused by Staphylococcus aureus. It has excellant tissue penetration, it accumulates in abscesses, it is not impeded by high bacterial burden at the infection site and no renal dose adjustments are needed. Good oral absorption makes it an important option in outpatient therapy as follow up after intravenous therapy. CL is also a good alternative antibiotic for the penicillin allergic patients and in infections due to MRSA.CL is less costly than some of the newer agents that might be considered for these infections. However, recent reports indicate that treatment failure may occur in the case of inducible MLSB resistance, is spite of invitro susceptibility to CL [6].

Accurate susceptibility data are important for appropriate therapy decisions. However, false in vitro susceptibility results may be obtained by the microdilution method and disc diffusion testing with erythromycin and clindamycin disc is nonadjacent positions. Hence, the routine testing of Staphylococcal isolates for inducible clindamycin resistance is recommended by the 2005 CLSI guidelines [7].

The prevalence of the MRSA strains was high (54.78\%) in the present study, which is similar to the results obtained by Gadepalli at al (52\%), Mallick SR et al (51.6\%) and S. Anuprabha et al (54.8\%) [7],[8],[9].

Of the total 230 isolates, $57(24.78 \%$ ) were of the iMLSB phenotype in the present study, which is similar to that reported by Angel et al from CMC Vellore (23.2\%) and Fiebelkorn et al who have reported 28\% iMLSB [10],[11].. Various authors have highlighted the relationship of MRSA and MSSA with different phenotypes of S.aureus isolates. 41 (32.5\%) of the 126 MRSA isolates were found to be of the iMLSB phenotype in the present study. Studies from different parts of India have reported 30\% to $64 \%$ of the MRSA isolates to be of the iMLSB phenotype [8].

In the present study, $15.53 \%$ of the MSSA isolates showed iMLSB resistance, which correlates with the findings by Delialiogu et al, who reported 10.7\% iMLSB resistance in the MSSA isolates and Gupta at al who reported $17.3 \%$ iMLSB resistance in the MSSA isolates [3][12].

In the present study, $42(18.26 \%)$ S.aureus strains were of the cMLSB type, of which $25.39 \%$ were MRSA and $9.6 \%$ were MSSA. Angel et al have not found any cMLSB resistance in the S.aureus strains. However, Gupta et al have reported $19 \%$ cMLB resistance, $46 \%$ of which were of the MRSA type and $10 \%$ were of the 
MSSA type [10][12]. 15.65\% S.aureus strains showed the MSB phenotype in the present study. Gadepalli et al have reported $12 \%$ strains of the MSB phenotype among the S.aureus strains [7].

Most of the studies have indicated a higher prevalence of constitutive resistance than inducible resistance in Staphylococcus aureus. The true incidence depends on the patient population studied, the geographical region, the hospital characteristics and Methicillin susceptibility. The present study showed a higher incidence of iMLSB (24.89\%) as compared to cMLSB $(18.26 \%)$ in the S.aureus isolates, which was similar to the findings of a study conducted by Mallick et al, who reported 18.6\% strains of iMLSB and 3.8\% strains of cMLSB [8].

Furthermore, the D-test was performed as a routine test on all Staphylococcal strains which were isolated in the laboratory, whereas most of the published D-test studies select only isolates that are ER resistant and CL susceptible for testing. The authors were concerned that if the D-test was delayed until ER resistance and CL susceptibility were noted in the isolate, the results may not be available for maximum clinical utility.

In conclusion, the implementation of the D-test or the Disc induction test, a simple, auxiliary method with routine antibiotic susceptibility testing, delineates inducible and constitutive clindamycin resistance. The high rates of occurrence of inducible resistance in both the MSSA and MRSA strains raise concerns that clindamycin treatment failures may occur with MSSA as well as with MRSA infection. Consequently, early detection helps in the use of CL only in infections caused by truly CL susceptible S.aureus and thus helps to avoid treatment failures.

\section{References}

[1] Lertcanawanichakul M, Chawawisit K, Choopan A, Nakbud K, Dawveerakul K. Incidence of constitutive and inducible clindamycin resistance in clinical isolates of methicillin resistant Staphylococcus aureus. Walailak J Sci \&Tech 2007;4:155-63.

[2] Yilmaz G,Aydin K, Iskender S, Caylan R, Koksal I. Detection and prevalence of inducible resistance in Staphylococci. J Med Microbiol 2007; 56:342-5.

[3] Delialioglu N, Aslan G, Ozturk C, Baki V, Sen S, Emekdas G.Inducible clindamycin resistance in Staphylococci isolated from clinical samples. Jpn J Infect Dis 2005; 58:104-6.

[4] Mandell, Douglas, and Bennett's Principles and Practice of Infectious Diseases, 7th ed.

[5] Clinical and laboratory standards institute (CLSI).performance standards for antimicrobial disk susceptibility tests. Approved standard M2-A7, 11 th ed.2005: 768-800.

[6] Chelae S, Laohaprertthisarn V, Phengmak M, Kongmuang U, Kalnauwakul S.Detection of inducible clindamycin resistance in Staphylococci by disc diffusion induction test. J Med Assoc Thai 2009; 92(7):947-51.

[7] Gadepalli R, Dhawan B ,Mohanty S, Kapil A, Das BK, Chaudhary R.Inducible clindamycin resistance in clinical isolates of Staphylococcus aureus.Indian J Med Res 2006;123:571-3.

[8] Mallick SK, Basak S, Bose S. Inducible clindamycin resistance in Staphylococcus aureus-A therapeutic challenge. Journal of clinical and diagnostic research 2009; 3:1513-18

[9] Anuprabha S., Sen MR, Nath G, Sharma BM, Gulati Ak, Mohapatra TM. Prevalence of Methicillin Resistant Staphylococcus aureus in a tertiary referral hospital in Eastern Utter Pradesh. Indian J.Med. Microbiol 2003;90

[10] Angel MR, Balaji V, Prakash JAJ, Brahmadathan KN, Mathews MS.Prevalence of inducible clindamycin resistance in gram positive organisms in a tertiary care centre.Indian J Med Microbiol 2008;26:262-4.

[11] Fiebelkorn KR, Crawford SA, McElmeel ML, Jorgensen JH. Practical disk diffusion method for detection of inducible clindamycin resistance in Staphylococcus aureus and Coagulase negative Staphylococci. J Clin Microbiol 2003; 41:4740-4.

[12] Gupta V, Datta P, Rani H, Chander J. Inducible clindamycin resistance in Staphylococcus aureus: A study from North India. J Postgrad Med 2009; 55:176-9 